\title{
ÁCIDO ASCÓRBICO NO DILUIDOR PARA CRIOPRESERVAÇÃO DE SÊMEN CAPRINO
}

\section{ASCORBIC ACID IN EXTENDER FOR CRYOPRESERVATION OF GOAT SEMEN}

\author{
Lindomar Sousa Brito ${ }^{1^{*}}$ \\ Larissa Pires Barbosa ${ }^{1}$ \\ Max Vitória Resende ${ }^{2}$ \\ Alexandre Moraes Pinheiro ${ }^{1}$ \\ Caline Santana da França ${ }^{1}$ \\ Reuber de Carvalho Cardoso ${ }^{1}$ \\ ${ }^{1}$ Universidade Federal do Recôncavo Baiano, Cruz das Almas, BA, Brasil. \\ 2Universidade de Salvador - UNIFACS, Salvador, BA, Brasil. \\ *Autor para correspondência - lindomar.britto@gmail.com
}

\section{Resumo}

Objetivou-se determinar o efeito e o melhor nível de inclusão de ácido ascórbico em meio diluidor TRIS-Gema para criopreservação de sêmen caprino. Foram utilizados cinco ejaculados de três bodes da raça Anglo Nubiana. Cada ejaculado foi dividido em quatro alíquotas de $200 \mu \mathrm{L}$, compondo quatro tratamentos: um sem adição de ácido ascórbico (controle) e os demais com 0,0528; 0,1056 e 0,1584mg/mL de ácido ascórbico no diluidor TRIS-Gema. Avaliaram-se motilidade espermática progressiva e vigor espermático pós-diluição, pós-resfriamento e pós-descongelamento; integridade de membrana (HOST); integridade acrossomal e teste de termorresistência lento (TTR). Os dados foram submetidos à Análise de Regressão a 5\% de probabilidade. Não houve diferença para motilidade progressiva e para vigor espermático, repectivamente, na pós-diluição; pós-resfriamento e pós-descongelamento, assim como para os testes complementares HOST e após 120 minutos do TTR, para motilidade e vigor, no pós-descongelamento ( $\mathrm{P}>0,05)$. Houve comportamento quadrático com a inclusão do ácido ascórbico para integridade acrossomal (61,58\%), com nível ótimo de 0,1006mg/mL de ácido ascórbico $(\mathrm{P}<0,05)$. A inclusão de $0,1006 \mathrm{mg} / \mathrm{mL}$ de ácido ascórbico no diluidor TRIS-Gema melhorou a integridade acrossomal durante o processo de criopreservação, podendo ser uma alternativa na composição dos diluidores seminais caprinos.

Palavras-chave: antioxidantes; espermatozoide; estresse oxidativo; TRIS-gema.

\section{Abstract}

The objective of this study was to determine the optimal inclusion level of ascorbic acid in TRISyolk extenders for cryopreservation of goat semen. We used five ejaculates of three Anglo Nubian goats. Semen from each animal was divided into four $200 \mu \mathrm{L}$ aliquots, composing four treatments: no 
addition of ascorbic acid (control) and $0.0528,0.1056$, and $0.1584 \mathrm{mg} / \mathrm{mL}$ of ascorbic acid in TRISegg yolk. We evaluated motility and sperm vigor after dilution, post-cooling, and post-thawing; membrane integrity (HOST); acrosomal integrity, and slow thermoresistance test (TRT). Data were subjected to regression analysis at 5\% probability. There was no difference for the progressive motility and sperm vigor, respectively, in the post-dilution, after cooling and post-thawing, as well as the additional tests HOST and after 120 minutes of TRT, for motility and force, respectively, in postthawing $(\mathrm{P}>0.05)$. There was a quadratic effect with the addition of ascorbic acid to the acrosome integrity parameter, presenting a great maximum level of $0.1006 \mathrm{mg} / \mathrm{mL}$ ascorbic acid for acrosome integrity of $61.58 \%(\mathrm{P}<0.05)$. The addition of ascorbic acid was effective in maintaining sperm viability, especially acrosome integrity during the cryopreservation process until the level of 0.1006 $\mathrm{mg} / \mathrm{mL}$, and it may be an alternative composition of seminal extenders for goats.

Keywords: antioxidants; oxidative stress; sperm; TRIS-yolk.

Recebido em: 06 março de 2016

Aceito em: 31 julho de 2017

\section{Introdução}

A resistência espermática durante a criopreservação varia entre machos de uma mesma espécie, assim como em machos de diferentes espécies. Em geral, os espermatozoides de pequenos ruminantes são mais sensíveis à criopreservação em comparação com a espécie bovina ${ }^{(1)}$. Além dessas características intrínsecas observadas nas diferentes espécies durante o processo de criopreservação, o choque térmico é normalmente associado ao estresse oxidativo, devido à geração de espécies reativas ao oxigênio (ROS), pelos espermatozoides mortos e oxigênio atmosférico. Esse fato promove um desequilíbrio entre o efeito protetor dos antioxidantes enzimáticos do plasma seminal desempenhado pelo superóxido dismutase (SOD), pela catalase (CAT), pela glutationa peroxidase (GSH-Px) e pela produção de ROS, que estão envolvidos com os danos causados à membrana plasmática e ao DNA espermático ${ }^{(2,3)}$.

Nesse aspecto, o uso de antioxidantes no diluidor seminal pode melhorar a eficácia contra o dano peroxidativo e a congelabilidade seminal, visto que o aumento da geração de ROS, principalmente em amostras manipuladas in vitro, diminui as defesas antioxidantes ${ }^{(3)}$. Pesquisadores têm testado diversas substâncias antioxidantes no diluidor seminal, incluindo o ácido ascórbico, para as espécies ovina $^{(4)}$, bovina ${ }^{(5)}$ e caprina ${ }^{(6)}$. No entanto, a dosagem desse antioxidante no diluidor seminal ainda é controversa, uma vez que observou-se redução na motilidade espermática no pós-descongelamento, quando houve adição de ácido ascórbico nas concentrações entre 5 e $10 \mathrm{mg} / \mathrm{mL}^{(7)}$ no sêmen de ovino, ao passo que concentrações inferiores dessa substância $(600 \mu \mathrm{M} / \mathrm{L})$ não apresentaram o mesmo comportamento $^{(4)}$.

Dessa forma, este estudo teve como objetivo determinar o efeito e o melhor nível de inclusão de ácido ascórbico no meio diluidor TRIS-Gema para criopreservação de sêmen caprino. 


\section{Material e Métodos}

Este trabalho foi previamente avaliado e aprovado pelo Comitê de Ética da Universidade Federal do Recôncavo da Bahia (CEUA - UFRB), processo 23007.005461/2014-18, atendendo aos princípios éticos e do bem estar animal.

O experimento foi realizado na cidade de Cruz das Almas-BA, localizada no paralelo $12^{\circ} 39^{\prime} 54^{\prime \prime}$, latitude Sul e meridiano 39 $04^{\prime} 44^{\prime \prime}$, longitude Oeste; no período de verão, entre fevereiro e março de 2014. Utilizaram-se três reprodutores da raça Anglo Nubiana, entre dois e três anos de idade, clinicamente sadios e aptos para reprodução, segundo as normas de exame andrológico do Colégio Brasileiro de Reprodução Animal ${ }^{(9)}$. Os animais foram mantidos em regime semi-intensivo de produção, com acesso à pastagem de Brachiaria (Brachiaria decumbens), suplementados com concentrado protéico (800g/animal/dia - Nutrividas®), sal mineral e água ad libitum.

As coletas de sêmen foram realizadas na frequência de duas vezes por semana, pelo método de vagina artificial, na presença de uma cabra em estro como manequim, totalizando cinco coletas viáveis por animal. Após as coletas, os ejaculados foram encaminhados ao laboratório e acondicionados em banho-maria a $32{ }^{\circ} \mathrm{C}$. Em seguida, avaliou-se o volume, a cor, o turbilhão, a motilidade progressiva e o vigor, bem como a concentração, a morfologia espermática e a integridade de membrana dos espermatozoides. Apenas as amostras que atenderam ao padrão mínimo preconizado pelo CBRA ${ }^{(9)}$ foram encaminhadas à criopreservação.

Após avaliação, cada ejaculado foi dividido em quatro alíquotas de $200 \mu \mathrm{L}$ e diluído em meio diluidor TRIS-Gema ${ }^{(10)}$, compondo quatro tratamentos, sendo: sem adição de ácido ascórbico (controle) e com 0,0528; 0,1056 e 0,1584 mg/mL de ácido ascórbico, segundo Peixoto et al. ${ }^{(4)}$. As amostras foram diluídas para manter uma concentração espermática de aproximadamente 100-120 milhões por dose.

A criopreservação iniciou-se com a curva de resfriamento, que apresentou uma queda de $0,2{ }^{\circ} \mathrm{C} / \mathrm{min}$, atingindo o equilíbrio a $5{ }^{\circ} \mathrm{C}$ em 3 horas. Para tal, os tubos com o sêmen diluído foram colocados em canecas de alumínio, contendo $300 \mathrm{~mL}$ de água a $32{ }^{\circ} \mathrm{C}$, acondicionadas em caixa isotérmica de poliestireno expandido de $11 \mathrm{~L}$, preenchida por cubos de gelo. Em seguida, para realizar o congelamento, o sêmen foi envasado em palhetas de $0,25 \mathrm{~mL}$, seladas com massa de modelar atóxica, acomodadas em um suporte na posição horizontal a $5 \mathrm{~cm}$ do nitrogênio líquido durante 15 minutos, seguindo-se da imersão em nitrogênio líquido e armazenamento em botijão criogênico.

A motilidade espermática progressiva e o vigor espermático de cada partida foram analisados pósdiluição, pós-resfriamento e pós-descongelamento em microscópio de luz com contraste de fase, em aumento de 20 a 40X (Nikon E200). As avaliações de integridade de membrana plasmática (HOST) foram realizadas segundo Bittencourt et al. ${ }^{(11)}$, enquanto que as de integridade acrossomal foram feitas segundo Pope et al. ${ }^{(12)}$ e o teste de termorresistência lento (TTR) foi realizado segundo o CBRA ${ }^{(9)}$.

O delineamento experimental utilizado foi o inteiramente casualizado, com quatro tratamentos e 
quinze repetições. Para análise estatística dos resultados, utilizou-se o comando PROC GLM (do programa estatístico SAS 9.1®) e os dados foram submetidos à análise de regressão, adotando-se o nível de significância de $5 \%$. Contrastes polinomiais foram utilizados para se determinar o efeito linear e quadrático dos tratamentos.

\section{Resultados e Discussão}

Não houve diferença $(\mathrm{P}>0,05)$ entre os tratamentos quanto à motilidade espermática progressiva e ao vigor espermático pós-diluição, pós-resfriamento e pós-descongelamento (Tabela 1). Em todos os tratamentos, os valores de motilidade e de vigor pós-descongelamento se mantiveram acima dos limites mínimos preconizados para a espécie caprina (30\% para motilidade e 2 para vigor), segundo o CBRA $^{(9)}$, demonstrando eficiência no processo de criopreservação e dos diluidores utilizados, independente da adição do ácido ascórbico.

Tabela 1. Motilidade espermática progressiva e vigor espermático pós-diluição, pósresfriamento e pós-descongelamento de sêmen diluído em meio acrescido de ácido ascórbico

\begin{tabular}{|c|c|c|c|c|c|c|c|}
\hline \multirow{2}{*}{ Item } & \multicolumn{4}{|c|}{ Níveis de inclusão $(\mathrm{mg} / \mathrm{mL})$} & \multirow{2}{*}{$\mathbf{E P M}^{1}$} & \multicolumn{2}{|c|}{ Significância } \\
\hline & 0 & 0,0528 & 0,1056 & 0,1584 & & $\operatorname{Lin}^{2}$ & Quad $^{3}$ \\
\hline \multicolumn{8}{|l|}{ Motilidade (\%) } \\
\hline Pós-Diluição & 90,3 & 90,3 & 89,7 & 90,0 & 0,82 & 0,653 & 0,840 \\
\hline Pós-Resfriamento & 84,3 & 82,0 & 83,0 & 84,3 & 0,02 & 0,895 & 0,279 \\
\hline Pós-Descongelamento & 72,7 & 71,7 & 73,3 & 73,0 & 1,65 & 0,752 & 0,860 \\
\hline \multicolumn{8}{|l|}{ Vigor (0 a 5) } \\
\hline Pós-Diluição & 4,9 & 5,0 & 5,0 & 5,0 & 0,10 & 0,185 & 0,322 \\
\hline Pós-Resfriamento & 4,6 & 4,6 & 4,6 & 4,7 & 1,87 & 0,551 & 0,739 \\
\hline Pós-Descongelamento & 4,3 & 4,1 & 4,3 & 4,2 & 0,10 & 0,765 & 0,739 \\
\hline
\end{tabular}

${ }^{1}$ Erro Padrão da Média (\%), ${ }^{2}$ Significância para efeito linear, ${ }^{3}$ Significância para efeito quadrático.

*Os dados foram analisados por regressão a $5 \%$ de probabilidade.

A ausência de efeito da adição de ácido ascórbico na congelabilidade seminal pode ter sido devido à quantidade de ácido arcórbico utilizada, não sendo necessária para manter o sistema antioxidativo eficiente e, por conseguinte, não tendo eficiência no combate ao dano oxidativo. Peixoto et al. ${ }^{(4)}$ encontraram resultados similares na espécie ovina, de modo que a concentração máxima de 0,1056 $\mathrm{mg} / \mathrm{mL}$ de ácido ascórbico no diluidor seminal Tris-Gema também não proporcionou melhorias 
significativas na motilidade progressiva e no vigor espermático no pós-descongelamento.

Estudos mais recentes mostram resultados positivos na motilidade espermática de caprinos com adição de $8,5 \mathrm{mg} / \mathrm{mL}$ de ácido ascórbico no diluidor seminal ${ }^{(8)}$, ao passo que outros resultados demonstraram que a inclusão de ácido ascórbico em até $4,5 \mathrm{mg} / \mathrm{mL}$ resultaram num aumento significativo da motilidade espermática progressiva, de modo que concentrações mais elevadas (10 $\mathrm{mg} / \mathrm{mL}$ ) conferiram uma taxa menor de motilidade dos espermatozoides de bovinos ${ }^{(5)}$ e ovinos $^{(7)}$. Portanto, resultados divergentes com o uso de antioxidantes são comuns, pois podem estar relacionados ao tipo de antioxidante, ao meio diluidor, à concentração do antioxidante e à espécie $\operatorname{animal}^{(5,7,13,14)}$.

Não houve efeito da inclusão de ácido ascórbico no diluidor de sêmen caprino na integridade de membrana plasmática ( $\mathrm{P}>0,05)$ (Tabela 2). Baseado nos resultados médios de motilidade e de vigor, esperava-se haver uma maior proporção de células com membrana íntegra nos tratamentos com diluidor com ácido ascórbico.

Tabela 2. Integridade acrossomal e HOST de sêmen caprino criopreservado em diluidor acrescido de ácido ascórbico

\begin{tabular}{lccccccc}
\hline \multirow{2}{*}{ Item } & \multicolumn{4}{c}{ Niveis de inclusão $(\mathbf{m g} / \mathbf{m L})$} & \multirow{2}{*}{ EPM $^{\mathbf{1}}$} & \multicolumn{2}{c}{ Significância } \\
\cline { 2 - 4 } & $\mathbf{0}$ & $\mathbf{0 , 0 5 2 8}$ & $\mathbf{0 , 1 0 5 6}$ & $\mathbf{0 , 1 5 8 4}$ & & $\mathbf{L i n}^{\mathbf{2}}$ & $\mathbf{Q u a d}^{\mathbf{3}}$ \\
\hline HOST (\%) & 50,6 & 53,9 & 52,8 & 52,1 & 2,81 & 0,823 & 0,578 \\
Int. Acrossomal (\%) & 50,0 & 60,2 & 60,4 & 58,3 & 3,51 & 0,053 & 0,034 \\
\hline
\end{tabular}

Equações de regressão

Int. Acrossomal $\quad * \hat{\mathrm{Y}}=-1106 \mathrm{x}^{2}+222,54 \mathrm{x}+50,383\left(\mathrm{R}^{2}=0,9513\right)$

${ }^{1}$ Erro Padrão da Média (\%), ${ }^{2}$ Significância para efeito linear, ${ }^{3}$ Significância para efeito quadrático.

*Os dados foram avaliados por meio de regressão a $5 \%$ de probabilidade.

Esse comportamento corrobora o pressuposto de que as lesões da membrana podem não estar associadas à perda da motilidade espermática ${ }^{(15)}$. É possível inferir, a partir dessas diferenças, que a cinética espermática não depende exclusivamente do transporte de elementos através da membrana e, sim, de outros mecanismos bioquímicos.

Diante desse contexto, vale destacar a importância em avaliar a integridade da membrana espermática, visto que a criopreservação confere danos irreversíveis aos espermatozoides. Essas injúrias causadas aos espermatozóides se devem, principalmente, ao choque térmico, à exposição ao oxigênio durante a manipulação do sêmen, ao estresse osmótico, à toxicidade dos crioprotetores, à formação e à dissolução dos cristais no ambiente extracelular ${ }^{(16)}$.

Além disso, concentrações elevadas de ácido ascórbico podem alterar as características da membrana plasmática dos espermatozoides e reduzir a peroxidação lipídica (LPO) ao ponto de afetar a sua fluidez ${ }^{(5)}$. Por outro lado, a presença do antioxidante em baixas quantidades contribui para a produção fisiológica de ROS, que são essenciais para as alterações nas membranas no momento da 
capacitação ${ }^{(17)}$. Wright et al. ${ }^{(18)}$ afirmaram que a LPO ocorre quando as ligações duplas de ácidos graxos poli-insaturados são atacadas pelas ROS, criando um radical peróxido lipídico. Assim, há uma propagação para as moléculas de ácidos graxos vizinhas, reação em cadeia, causando sérios danos às membranas lipídicas ${ }^{(19)}$.

Houve efeito quadrático negativo com a inclusão do ácido ascórbico para a variável integridade acrossomal, em função do aumento de antioxidante no diluidor, apresentando um nível ótimo de 0,1006 mg/mL de ácido ascórbico para uma integridade acrossomal de 61,58\% $(\mathrm{P}<0,05)$ (Tabela 2 e Figura 1).

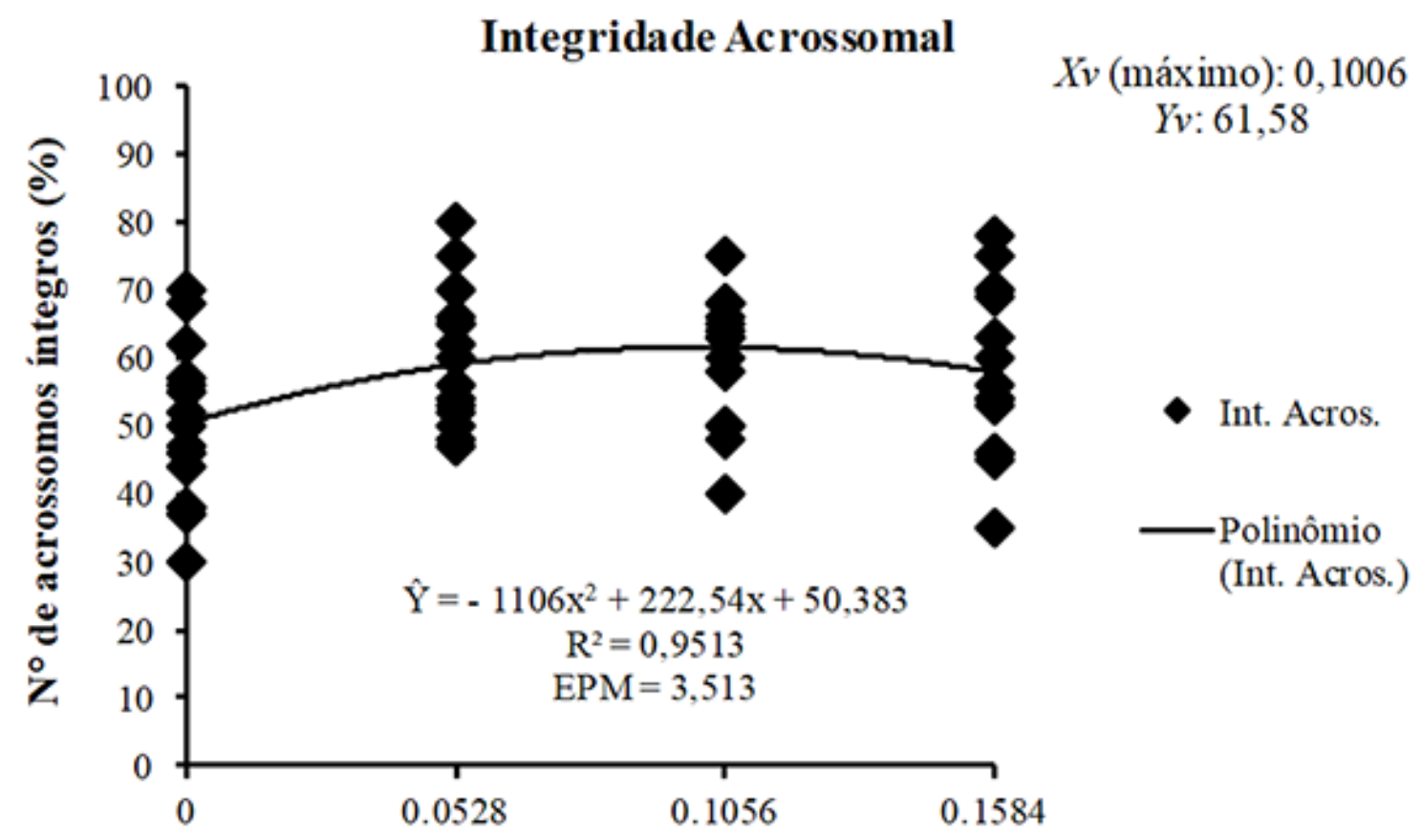

\section{Níveis de inclusão de ácido ascórbico $(\mathrm{mg} / \mathrm{mL})$}

Figura 1. Percentagem de acrossomos intactos de sêmen diluído com meio acrescido com níveis de ácido ascórbico pós-descongelamento.

Nesse aspecto, a inclusão de ácido ascórbico no diluidor seminal manteve a viabilidade espermática até o nível de 0,1006 mg/mL, possivelmente em virtude da redução dos efeitos deletérios de ROS, visto que houve uma maior percentagem de espermatozoides com membrana acrossomal íntegra. Esses achados são importantes no intuito de viabilizar a fertilização in vivo e in vitro (FIV), uma vez que a inclusão de ácido ascórbico no diluidor contribuiu para um percentual superior de espermatozoides vivos com acrossoma intacto em relação ao grupo controle, corroborando os achados de Peixoto et al. ${ }^{(4)}$, os quais afirmaram que concentrações inferiores dessa substância proporcionam melhorias significativas na qualidade espermática.

Esses mecanismos estão relacionados com o aumento da ação antioxidante que, além de atuar de forma natural no controle da ação das ROS, auxilia vários outros mecanismos, incluindo a redução da LPO durante o processo de criopreservação e, assim, protegem a membrana acrossomal ${ }^{(20,5)}$. Isso ocorre porque o ácido ascórbico atua na proteção do DNA espermático, mais precisamente no 
citocromo P-450, fato que impede a destruição da membrana por pseudo-substratos oxidativos e oxigênio $^{(21,22)}$, evidenciando a alta eficiência do antioxidante na contenção dos danos oxidativos provocados pela criopreservação do sêmen de caprinos.

É importante considerar que, assim como as células somáticas, os espermatozoides produzem pequenas quantidades de ROS como um subproduto da cadeia de transferência de elétrons na mitocôndria ${ }^{(23)}$. Isso acarreta uma maior quantidade de ROS na célula, consequentemente, mais danos ao DNA mitocondrial, indicando que, quanto mais células com mitocôndria sem atividade, menor a produção de ATP e fornecimento de energia para a motilidade espermática, sendo uma das possíveis causas de infertilidade em machos ${ }^{(24)}$. Por esse motivo, é importante que o acrossomo esteja íntegro, um pré-requisito essencial para a ligação do espermatozoide à zona pelúcida e para a reação acrossomal subsequente $\mathrm{e}^{(25)}$.

Ao final de duas horas de incubação, não foram encontradas diferenças para o TTR $(P>0,05)$ para as variáveis motilidade espermática progressiva e vigor espermático (Tabela 3).

Tabela 3. Motilidade espermática progressiva (\%) e vigor espermático do sêmen caprino descongelado em meio diluidor contendo ácido ascórbico e submetido ao teste de termorresistência

\begin{tabular}{|c|c|c|c|c|c|c|c|}
\hline \multirow{2}{*}{ Item } & \multicolumn{4}{|c|}{ Níveis de inclusão $(\mathrm{mg} / \mathrm{mL})$} & \multirow{2}{*}{$\mathbf{E P M}^{\mathbf{1}}$} & \multicolumn{2}{|c|}{ Significância } \\
\hline & 0 & 0,0528 & 0,1056 & 0,1584 & & $\operatorname{Lin}^{2}$ & Quad $^{3}$ \\
\hline \multicolumn{8}{|c|}{ Motilidade (\%) } \\
\hline $0 \mathrm{~min}$ & 73,7 & 72,3 & 74,0 & 73,7 & 1,867 & & \\
\hline $5 \mathrm{~min}$ & 72,3 & 70,7 & 72,7 & 72,3 & 2,002 & & \\
\hline $30 \mathrm{~min}$ & 67,7 & 65,3 & 69,0 & 67,3 & 1,953 & 0,572 & 0,711 \\
\hline $60 \mathrm{~min}$ & 62,0 & 59,0 & 64,7 & 62,0 & 2,204 & & \\
\hline $120 \mathrm{~min}$ & 47,0 & 45,3 & 48,0 & 49,3 & 4,424 & & \\
\hline \multicolumn{8}{|l|}{ Vigor } \\
\hline $0 \min$ & 4,3 & 4,1 & 4,3 & 4,2 & 0,099 & & \\
\hline $5 \mathrm{~min}$ & 4,3 & 4,1 & 4,2 & 4,2 & 0,095 & & \\
\hline $30 \mathrm{~min}$ & 4,0 & 4,0 & 4,2 & 4,0 & 0,097 & 0,896 & 0,964 \\
\hline $60 \mathrm{~min}$ & 3,7 & 3,7 & 3,8 & 3,7 & 0,134 & & \\
\hline $120 \mathrm{~min}$ & 3,1 & 3,0 & 3,0 & 3,1 & 0,166 & & \\
\hline
\end{tabular}

'Erro Padrão da Média (\%), ${ }^{2}$ Significância para efeito linear, ${ }^{3}$ Significância para efeito quadrático.

* Os dados foram avaliados por meio de regressão a $5 \%$ de probabilidade.

Apesar de não ter observado significância para essas variáveis, é importante considerar que o comportamento durante o teste de termorresistência (TTR) foi semelhante para ambas, sendo altas nas primeiras horas do teste, diminuindo nas horas intermediárias e, posteriormente, estabilizando em baixos valores nas horas finais do teste ${ }^{(26)}$. Isso ocorre porque as reservas energéticas vão se esgotando, em virtude de o metabolismo celular estar elevado. Assim, há um aumento considerável de espermatozoides mortos e, por conseguinte, maior produção de ROS, mais danos peroxidativos e menor viabilidade espermática. 
Maxwell e Watson ${ }^{(27)}$ afirmaram que a geração de espécies reativas ao oxigênio é consequência normal do metabolismo oxidativo e, possivelmente, elas devem estar associadas aos danos espermáticos em condições hipotérmicas de estocagem. Além disso, diversos fatores podem atuar sobre as células espermáticas, tais como alterações na composição, $\mathrm{pH}$, temperatura e osmolaridade do meio em que as circunda, provocando alterações irreversíveis em suas membranas ${ }^{(16)}$.

Nagy et al. ${ }^{(28)}$ demonstraram que o percentual de espermatozoides vivos de touros com acrossoma intacto diminui ao serem incubados a $37^{\circ} \mathrm{C}$ por um período de $4 \mathrm{~h}$, pois o estresse térmico provoca alterações na membrana plasmática e acrossomal. Portanto, esse processo é fundamental para se predizer a longevidade e a resistência espermática do sêmen caprino durante a passagem pelo trato genital da fêmea, etapa essencial antes da penetração nas células do cumulus e na zona pelúcida.

\section{Conclusão}

A inclusão de 0,1006 mg/mL de ácido ascórbico no diluidor TRIS-Gema melhorou a integridade acrossomal durante o processo de criopreservação, podendo ser uma alternativa na composição dos diluidores seminais de caprinos.

\section{Referências}

1. Küçük N, Aksoy M, Uçan U, Ahmad E, Naseer Z, Ceylan A, Serin I. Comparison of two different cryopreservation protocols for freezing goat semen. Cryobiology. 2014;68:327-331. http://dx.doi.org/10.1016/j.cryobiol.2014.04.009.

2. Bucak MN, Tuncer PB, Sariozkan S, Ulutas PA, Coyan K, Baspinar N, Ozkalp B. Effects of hypotaurine, cysteamine and aminoacids solution on post-thaw microscopic and oxidative stress parameters of Angora goat semen. Res Vet Sci. 2009;87:468-72. doi:10.1016/j.smallrumres.2008.11.011.

3. Peris SI, Bilodeau JF, Dufour M. Impact of Cryopreservation and reactive oxygen species on DNA inetgrity, lipid peroxidation, and functional parameters in ram sperm. Mol Reprod Dev. 2007;74:878-892. doi: 10.1002/mrd.20686.

4. Peixoto ALVA, Monteiro Jr. PLJ, Câmara DR, Valença RMB, Silva KMG, Guerra MMP. Efeito do tempo de incubação pós-descongelação sobre a viabilidade de espermatozoides ovinos criopreservados com tris-gema suplementado com vitamina C e Trolox. Cienc Vet Trop. 2008;11:16-24. Disponível em: $<$ http://www.rcvt.org.br/volumes2.htm>. Acesso em: 06 jun de 2013.

5. Hu J H, Tian WQ, Zhao XL, Zan LS, Wang H, Li QW, Xin YP. The cryoprotective effects of ascorbic acid supplementation on bovine semen quality. Anim Reprod Sci. 2010;121:72-77. doi: 10.1016/j.anireprosci.2010.04.180.

6. Gangwar C, Kharche SD, Ranjan R, Kumar S, Goel AK, Jindal SK, Agarwal SK. Effect of vitamin C supplementation on freezability of Barbari buck semen. Small Ruminant Research. 2015;129:104-107. Disponível em: <http://dx.doi.org/10.1016/j.smallrumres.2015.06.002>. Acesso em: 27 jul de 2017. 
7. Sönmez M, Demirci E. The Effect of Ascorbic Acid on the Freezability of Ram Semen Diluted with Extenders Containing Different Proportions of Glycerol. Turk J Vet Anim Sci. 2004;28:893-899. Disponível em: < http://journals.tubitak.gov.tr/veterinary/index.htm>. Acesso em: : 06 jun de 2013.

8. Memon AA, Wahid H, Rosnina Y, Goh YM, Ebrahimi M, Nadia FM. Effect of antioxidants on post thaw microscopic, oxidative stress parameter and fertility of Boer goat spermatozoa in Tris egg yolk glycerol extender. Anim Reprod Sci. 2012;136:55-60. http://dx.doi.org/10.1016/j.anireprosci.2012.10.020.

9. CBRA (COLÉGIO BRASILEIRO REPRODUÇÃO ANIMAL). Manual para exame andrológico e avaliação de sêmen animal. $3^{\text {a }}$ ed. Belo Horizonte: CBRA, 2013. 104p. Portuguese.

10. Resende J, Weitze KF. Congelamento de sêmen de caprino com diluente tris modificado. In: REUNIÃO ANUAL DA SOCIEDADE BRASILEIRA DE ZOOTECNIA, 24, 1987, Brasília. Anais... Brasília: Soc Bras Zootec. 1987, p. 381.

11. Bittencourt RF, Ribeiro Filho, AL, Santos ADF, Chalhoub M, Alves SGG, Vasconcelos MF, Leandro EES, Guimarães JD. Utilização do teste hiposmótico para avaliar a eficácia de diferentes protocolos de criopreservação do sêmen caprino. Cienc Anim Bras. 2005;6:213-218. Disponível em: < http://www.revistas.ufg.br/index.php/vet/article/view/362>. Acesso em: 12 ago. 2013.

12. Pope CE, Zhang YZ, Dresser BL. A simple staining method for valuating acrosomal status of cat spermatozoa. J Zoo Wild Med. 1991;22:87-95.

13. Sikka SC. Role of oxidative stress and antioxidants in and rology and assisted reproductive technology. J. Androl. 2004;25:5-18. doi: 10.1002/j.1939-4640.2004.tb02751.x.

14. Satorre MM, Breininger E, Beconi MT, Beorlegui NB. $\alpha$-Tocopherol modifies tyrosine phosphorylation and capacitationlike state of cryopreserved porcine sperm. Theriogenology. 2007;68:958-965. doi: 10.1016/ j.theriogenology.2007.06.021.

15. Lin M, Morshedi M, Srisombut C, Nassar A, Oehninquer S. Plasma membrane integrity of cryopreserved human sperm: an investigation of the results of hypoosmotic swelling test, the water test, and cosin-Y staining. Fertil Steril. 1998;70:1148-1155. doi: 10.1016/S0015-0282(98)00351-3.

16. Watson PF. The causes of reduced fertility with cryopreserved semen. Anim Reprod Sci. 2000;60-61:481492. doi: 10.1016 / S0378-4320 (00) 00099-3.

17. De Lamirande E, Jiang H, Zini A, Kodama H, Gagnon C. Reactive oxygen species and sperm physiology. Rev. Reprod. 1997;2:48-54. Disponível em: < http://www.ncbi.nlm.nih.gov/pubmed/9414465>. Acesso em: 10 out. 2013.

18. Wright C, Milne S, Leeson H. Sperm DNA damage caused by oxidative stress: modifiable clinical, lifestyle and nutritional factors in male infertility. Reprod Biol Med Online. 2014;28:684-703. doi.org/10.1016/j.rbmo.2014.02.004.

19. Mylonas C, Kouretas D. Lipid peroxidation and tissue damage. I Vivo. 1999;13:295-309. Disponível em: <http://www.ncbi.nlm.nih.gov/pubmed/10459507>. Acesso em: 01 mai de 2014.

20. Knight JA, Blaylock RC, Searles DA. The effect of vitamins C and E on lipid peroxidation in stored erythrocytes. Ann. Clin. Lab. Sci. 1993;23:51-56. Disponível em: < http://www.ncbi.nlm.nih.gov/pubmed/8431001>. Acesso em: 08 set de 2014.

21. Fraga GG, Motchnik PA, Shigenaga MK, Helbrock JH, Jacob RA, Ames B. Ascorbic acid protects against endogenous oxidative DNA damage in human sperm. Proc Natl Acad Sci USA. 1991;88:11003-11006. Disponível em: < http://www.ncbi.nlm.nih.gov/pmc/articles/PMC53061/>. Acesso em: 06 set. 2014. 
22. Branco CS, Garcez ME, Pasqualotto FF, Erdtman B, Salvador M. Resveratrol and ascorbic acid prevent DNA damage induced by cryopreservation in human semen. Cryobiology. 2010;60:235-237. doi: 10.1016/j.cryobiol.2009.10.012.

23. Koppers AJ, De Iuliis GN, Finnie JM, Mclaughlin EA, Aitken RJ. Significance of mitochondrial reactive oxygen species in the generation of oxidative stress in spermatozoa. J. Clin. Endocrinol. Metab. 2008;93:31993207. doi: 10.1210/jc.2007-2616.

24. Shamsi MB, Kumar R, Bhatt A, Bamezai RN, Kumar R, Gupta NP, Das TK, Dada R. Mitochondrial DNA Mutations in etiopathogenesis of male infertility. Indian J. Urol. 2008;24:150-154. doi: 10.4103/0970$\underline{1591.40606 .}$

25. Verstegen J, Iguer-Ouada M, Onclin K. Computer assisted semen analyzer in andrology research and veterinary practice. Theriogenology. 2002;57:149-179. doi: 10.1016 / S0093-691X (01) 00664-1.

26. Kumar S. Cellular damages during cryopreservation and assessment of in vitro fertilizing capacity of spermatozoa. Ind Vet Med. J. 2000;24:1-6, 2000.

27. Maxwell WMC, Watson PF. Recent progress in the preservation of ram semen. Anim Reprod Sci. 1996;42:55-65. doi: 10.1016 / 0378-4320 (96) 01544-8.

28. Nagy S, Hallap T, Johannisson A, Martinez-Rodriguez H. Changes in plasma membrane and acrosome integrity of frozen-thawed bovine spermatozoa during a $4 \mathrm{~h}$ incubation as measured by multicolor flow cytometry. Anim. Reprod. Sci. 2004;80:225-235. doi: 10.1016/j.anireprosci.2003.08.003. 